\title{
THE VAGINAL SPECULUM: A REVIEW OF LITERATURE FOCUSING ON SPECULUM REDESIGNS AND IMPROVEMENTS TO THE PELVIC EXAM
}

\begin{abstract}
KELLY WONG, VICTORIA LAWTON
Abstract

We reviewed scientific literature relating to the vaginal speculum considering the widespread use of this tool within women's health. Through a literature review, it is clear that the speculum is not specialized enough to be used for all populations who require the procedure. Despite the fact that the current standard of care is not sufficient for all patients, evaluations of industry solutions are not evident in the current literature, explaining physician hesitancy to adopt these new devices. Additionally, while scholarly literature exists regarding overviews of the topic, novel designs, and general improvements for speculum usage in pelvic examinations, there are noticeable gaps in the scholarship regarding frequency of scholarly output and a blatant disregard for obese populations in vaginal speculum research. More scholarly literature must be published in order to improve awareness of the vaginal speculum and pelvic examinations so that women receive the best care possible. More specifically, novel designs must be evaluated for efficacy and comfort, and more research should be conducted on the pelvic examination procedure and its use on obese patients.
\end{abstract}

\section{Introduction}

7 he vaginal speculum is the staple instrument used to evaluate women's gynecological health. Its origins trace back to "the father of gynecology," J.

L Marion Sims, who developed the first designs on enslaved women in the 1840s through a series of "unsuccessful, unanesthetized surgeries" (Rossmann). Today's vaginal speculum has the same basic, bi-valve design as it did in its inception, with the most common designs being the Graves and Pederson specula (Rossmann).

Due to the nature of the design process and the vaginal speculum's lack of change in the past two centuries, while the speculum is functional, it is uncomfortable for the patient. Though novel specula have been developed, these redesigns (Kent; Yona; Mailach; Taylor; Bridea Medical; Traub; Matschukat; Duke) have not overtaken the traditional, bi-valve design developed by Sims. This could be attributed to the disconnect between academia and industry, as well as physician reluctance to use a design that hasn't been heavily tested (Taylor). Upon a literature review, it is clear that industry solution redesigns have not gone through enough of the rigorous testing required to overtake the current standard of care. Namely, only one redesign-the Veda Scope-is evident in the current literature. However, the Veda Scope is only evaluated for efficacy and comfort in two publications. No other industry solutions are evident in the literature.

The level of discomfort for the patient is of serious concern, as this is one of the factors that deter women from receiving necessary exams. Only $51 \%$ of women 


\section{Columbia Undergraduate Research Journal}

visit the gynecologist yearly (IPSOS). The CDC found that between 1988 and 2017 there has been a decrease in yearly gynecologist visits (Martinez). While this may be partially due to conflicting information about the necessity of yearly pelvic examinations, discomfort deters patients from getting these examinations (Bates) despite the fact that these exams test for cervical/vaginal cancer, sexually transmitted infections, and more (Taylor).

In addition, there is a concern regarding the lack of literature on the vaginal speculum following a literature review of the topic. Very few publications discussing the vaginal speculum were found despite the 20-year span and the use of several databases, with the last general overview of the speculum published in 2008 (Rossmann). This suggests that any issues arising from the usage of the vaginal speculum or updates to the procedure have gone unaddressed and unpublicized to the majority of the female population. The absence of a recent overview [1] of the function is especially concerning considering that 52 million pelvic examinations were performed in the United States in 2015 (U.S. Department of Health and Human Services).

This lack of research surrounding the speculum is even more scarce when considering obese populations. In fact, only five articles were found that addressed obese patients and the vaginal speculum, with the majority only tangentially related. The same issues that general patients face may be the same, or worse, for obese patients. In fact, physicians are often not equipped to handle obese patients during pelvic examinations, mostly due to the lack of larger specula, resulting in "delay, reluctance, or avoidance of medical visits" (Amy). If more information on how to manage care for this population existed, doctors would be better equipped to handle these situations both in demeanor and medical tools, and the yearly examination rates may increase along with earlier detection of the various diseases (Clarke; Friedman).

Through the literature review, three separate categories were chosen for the found publications: improvements to pelvic examinations, speculum redesigns, and miscellaneous. It is evident that the majority of research falls into the first category, which alters pelvic examination procedures rather than the speculum itself.

It is clear that the current standard of care-namely, using the Graves or Pederson speculum for pelvic examinations - is not sufficient for all populations who require the procedure. More specifically, it is not specific enough to be both effective and comfortable for all patients, especially for obese patients.

Additionally, it is clear that a lack of literature regarding the vaginal speculum exists. Despite scholarly literature providing insight on speculum overviews, redesigns, and improvements, there are noticeable gaps in the scholarship regarding frequency of scholarly output and a blatant disregard for obese populations in vaginal speculum research. More research must be conducted to evaluate the efficacy and comfort of industry redesigns, as well as vaginal speculum use in obese patients. 


\section{Methodology}

Google Scholar, PubMed, and Clinical Key Elsevier were searched between August 2020 and December 2020 to identify relevant articles. Search terms included "vaginal speculum," "vaginal speculum design," "vaginal speculum comfort," "redesign vaginal speculum," "vaginal speculum, women's health," "obesity, women's health," and "obese women, quality health care". Only articles published between January 1, 2000, and November 1, 2020, were included. Both peer-reviewed and nonpeer reviewed papers published in English were considered.



\section{Results}

Improvements to Pelvic Examinations

There were nine publications that focused on improving the pelvic examinations that were unrelated to a physical redesign of the vaginal speculum. Some 


\section{Columbia Undergraduate Research Journal}

publications targeted the same aspects of the pelvic exam, but most varied greatly in what they considered to be causes of discomfort or function during these examinations.

\section{Usage of Stirrups}

Two studies cited that the presence and usage of stirrups incited discomfort (Seehusen; Bates). One of the two publications tested this hypothesis on patients and concluded that the removal had received overall favorable results (Seehusen), while the other did not include any clinical trials but proposed the same conclusion (Bates). Seehusen et al examined the patient's physical comfort level, control of examination, and sense of vulnerability as variables in order to gauge the effect of the presence of stirrups during the examination. Overall, the usage of stirrups proved to be of particular salience to patient comfort with no detriment to the quality of examination. Bates et al also reference stirrup removal as a proponent to increasing patient comfort. However, despite the similar conclusions between both papers, the methodologies of the publications varied. Whereas Seehusen et al primarily focused on the impact of stirrups on patient comfort using clinical trials on a patient pool of 197 women, Bates et al was a list of recommendations with no primary data collected specifically supporting removal of stirrups. To clarify, Bates et al have specific citations from several studies that supported individual recommendation sections but did not investigate these problems using a clinical trial. Furthermore, although Bates et al recommended the removal of stirrups, it was noted that this suggestion was not advocated to be a significant component to improve patient comfort, unlike Seehusen et al. This could be the reason behind the difference of strength in advocacy regarding usage of stirrups during pelvic examination. Seehusen et al had more in-depth research regarding the removal of stirrups to improve patient comfort due to testing this hypothesis in a real setting compared to Bates et al, which was a list of recommendations to improve the pelvic examination in its entirety. Due to limited data from only two publications citing favorable results toward stirrup removal during pelvic examinations, it is not conclusive to argue for the complete removal of stirrups during examinations; however, it is a recommendation to research further.

\section{Addition of Sheaths}

Another point of improvement to the pelvic examination was the addition of sheaths to the vaginal speculum. Without changing other features of the vaginal speculum design, sheaths are slipped onto the speculum. Hill et al and Freeman targeted changing the visual aspect of the speculum to increase functionality and comfort for the patient but differed on type of improvements. Hill et al compared the standard Graves speculum with a standard Graves speculum covered with a 


\section{Columbia Undergraduate Research Journal}

transparent, polyurethane sheath in order to inhibit vaginal walls from caving in during examination. Visually, the polyurethane is stretched thin with holes spaced laterally along the side of the speculum and an opening cut at the end to allow for visualization of the cervix (Hill et al). Freeman advocated for a condom sleeve instead of a polyurethane sheath (Freeman). Both papers proposed flexible material as a way to combat vaginal tissue inhibiting the visibility of the cervix. Hill et al found that using a Graves speculum covered with a polyurethane sheath increased visibility of the cervix to $95.1 \%$. By comparison, Freeman did not conduct human-subject testing but proposed that a condom would increase visualization. This is corroborated by personal interviews with physicians who support condoms as a substitute sheath to enhance visualization of the cervix [Israel]. Hill et al's mean pain levels were lower using the sheathed speculum, but no statistically significant difference in comfort levels between the sheathed and unsheathed groups were found. Hill et al concludes that further studies should focus on comparing pain scores between the polyurethane sheath to the condom. Freeman made no arguments about improvements, other than adding a condom as a substitute sheath to the speculum. McDonagh et al, however, mentioned changing the visual aspect of the specula, but this was more of a redesign than a simple addition to the current design (McDonagh et al). McDonagh et al propose including a steel spring to ease strain in opening the speculum, as well as elongating the lower bill, which would be coated in heat-resistant silicone. Since McDonagh revises the design by changing the bill length and including a spring, the sheathed speculum would be considered a redesign rather than an addendum to current speculum. More research is needed to test sheaths on specula as a method of increasing comfort.

\section{Lubrication of Speculum}

Focusing on a separate aspect of the examination, Hill and Lamvu proposed that instead of a sheath to increase cervix visibility, the speculum should be coated in a lubricant gel to decrease discomfort prior to insertion. Results proved that there were significant decreases in pain scores among patients whose exams were conducted using lubricant gel compared to patients whose exams used only water as a lubricant. A visual analog scale was used as a metric to test patient comfort levels after the examination. This was the only publication found that conducted human-subject testing for lubrication on the vaginal speculum. Bates et al references water-based lubricant or lubricant that does not contain carbopol polymers as recommendations to improve the pelvic examination. These findings concur with Hill and Lamvu's result. McDonagh et al also mentions that current medical textbooks encourage water as a lubricant rather than a gel-based lubricant to ease speculum insertion. Although they imply lubricant usage, they do not explicitly promote it. Only two publications were found to support lubricant usage to improve patient comfort (McDonagh et al; Hill et al), but it is strongly recommended that lubricant be used to increase comfort. There 


\section{Columbia Undergraduate Research Journal}

were no disagreements between the papers, and this practice is often used among current physicians (Gates).

\section{General Improvements}

Other publications focused on more than one improvement to the pelvic exam. McDonagh et al and Bates et al varied in their listings of suggested improvements. McDonagh et al had comparatively less recommendations. The authors suggested the pelvic examination protocol and speculum design be revised and physician training should be updated. McDonagh et al suggests that physicians ought to increase patient involvement and camaraderie during the examination while following the Rad technique to decrease physical discomfort [2]. As referenced earlier, McDonagh et al continue that the vaginal speculum should have a longer lower bill, steel spring mechanism, and a sheath. In comparison, Bates et al have a longer list of recommendations which include having a chaperone during examinations, voiding urine immediately prior to exams, lubricating the speculum, and suggesting the removal of stirrups. The aspects proposed by both papers vary but have great overlap between them. This suggests that more research is needed to test the recommendations of both papers to test efficacy. This is to check whether this recommendation is worth pursuing if two papers are suggesting similar improvements. McDonagh et al make no population-directed specifications while Bates et al designate a section where providers vary their technique for specific populations, particularly obese women and transgender folk. There is a large 16-year gap in publishing timeframe between Bates et al and McDonagh et al. Yet, there is no discussion on whether the problems noted in Bates et al have been resolved or addressed in the McDonagh et al paper. It is clear that there is a disconnect between the publications, yet complications with the pelvic examination outlined in both papers are still pervasive in practice today with no active usage or testing of these recommendations.

\section{Self-insertion}

Wright et al proposes self-insertion of the speculum by patients to minimize emotional discomfort. Anxiety is often associated with the pelvic examination due to the patient's loss of autonomy over the process coupled with lack of general knowledge of the exam. Wright et al conducted a pilot study with clinical trials wherein women completed a self-questionnaire on physical and mental discomfort prior to the examination and the self-insertion technique was tested. A larger sample size was recommended for further research into feasibility of self-insertion, but overall, the results were favorable. $91 \%$ of the 133 women that partook in the study were significantly more at ease when self-inserting the speculum. Other publications made no mention of this revision to the examination, suggesting that there needs to be more 


\section{Columbia Undergraduate Research Journal}

research on feasibility of this method. There were mixed responses to self-insertion from physicians. Few stated that with guidance self-insertion of the speculum was possible, while the majority argued that without medical training, it is not reasonable for patients to self-insert the speculum (Israel). Self-insertion of the vaginal speculum is an uncommon technique, but further research would be necessary to determine if effectiveness of pelvic examinations would be adversely affected in exchange for comfort.

\section{Material and Situational Application}

Another improvement to the pelvic examination takes into account material and situational application of the speculum. Jones et al test the OfficeSpec, a sideopening plastic bi-valve speculum. The designs between the OfficeSpec and the bivalve vaginal speculum differ in material and inclusion of a built-in light. While there are current bi-valve speculua that share the same material, plastic, and built-in light, the OfficeSpec is the first plastic side-opening vaginal speculum, despite also being a fundamentally bi-valve design. The intention behind this study was to determine the most cost-effective speculum that would be used in austere environments where light and sanitary appliances are not readily available. Thus, comfort was not necessarily the highest priority in the study of this vaginal speculum. Jones et al focused on a separate sample population with women who were either affiliated with the military or hospital staff that would be using the speculum in areas of deployment. Results of the study concluded that the OfficeSpec was a practical and a viable option in austere environments where sanitary services and light are not readily available with deployment settings in mind. Although the study was not a conventional improvement to the pelvic examination, the situational use of the speculum in a military setting was a significant enough change to include in this section.

With entirely different intentions for improving the pelvic examination, Kunogi et al engineered a resin-based vaginal speculum applicator to ease the use of catheters in gynecologic interstitial brachytherapy [3] as an alternative to just using the current bi-valve design. The design is cylindrical in shape, made of two bills with multiple curved slits evenly interspersed on the outside of the applicator, and openings on both ends. There were no similarities in improvements shared between Kunogi et al and the other publications discussed. This can be attributed to the intention behind the design. The criterion for the applicator was to ease feeding catheters into the vaginal cavity - contrasting the goal of efficiency for the OfficeSpec and of comfort in other designs. The resin-based vaginal speculum applicator was engineered to cater to the Japanese female population in that the size of the speculum was made in mind of an average Japanese woman's vaginal cavity. Clinical trials were not conducted for this study, nor were physical vaginal models used to test the vaginal speculum applicator. Predictions for efficacy of the applicator were determined using 


\section{Columbia Undergraduate Research Journal}

measurements of an average Japanese woman's vaginal cavity with a computer program. Results concluded that the applicator is able to aid in dose distribution for gynecologic interstitial brachytherapy. We included this publication in this section due to the current bi-valve speculum remaining the same with the addition of an applicator due to specific circumstances.

\section{Specula Redesign}

In our search, we found four publications that focused on the efficacy of different speculum designs, while one of the publications advocated for a device to replace the speculum entirely. Two articles proposed their own design, whereas the other two studied existing specula, specifically the Veda Scope (Longmore; Thomas et al).

\section{Veda Scope}

The Veda Scope, a dilating vaginal speculum, was designed to increase patient comfort without inhibiting viewing of the cervix. Clinical trials in both publications served to compare physical comfort levels between the original steel speculum to the redesign (Longmore; Thomas et al). Longmore et al recruited healthy female participants 18 years old or older. Exclusion criteria included being pregnant, experiencing early postpartum, or having a recent miscarriage. Any medical or physical condition that could interfere with the study were also standards for exclusion, as well as any pap smears conducted within three months prior to the study. Participant's ages range from 18 years to 78 years with the mean age being 38.9 years. In comparison, the only inclusion criteria for Thomas et al was being a female age 18 who was not pregnant or early postpartum. Participant's ages ranged from 20 years to 65 years with the mean age of 39.2 years old. Both Longmore et al and Thomas et al were published in Australian/New Zealand journals. The origin of these studies could be the reason for the lack of publicity for the Veda Scope outside of both countries, as the Veda Scope is an Australian-founded device. Both studies were double blind in the type of speculum used for the cervix sampling to prevent bias in data. Both papers concluded favorable results with a majority of participants preferring the redesigned speculum, though Thomas et al notes that physicians would experience difficulty learning to use the device at first but would ease into it. Both Thomas et al and Longmore et al have concluded that the redesign was highly preferred over the current bi-valve speculum with no detriment to quality of cervix sample taken.

\section{McDonagh et al}

As previously mentioned, McDonagh et al recommended a separate design with silicone bills, a longer bottom bill, and a spring to ease the strain of propping up 


\section{Columbia Undergraduate Research Journal}

vaginal walls, therefore increasing patient physical comfort. The purpose behind this redesign is similar to the Veda Scope, since they also focus on patient physical comfort. The designs differ greatly in that the Veda Scope has an additional device to gradually dilate the vaginal cavity through inserting air. In contrast, McDonagh et al's design makes only slight modifications to the current bi-valve speculum. Both account for decreasing physical discomfort; however, the Veda Scope proposes more drastic changes compared to McDonagh et al. Since there is debate over which design would be more suitable in increasing patient physical comfort, further research with clinical trials ought to be conducted.

\section{Diokno et al}

Similar to how Kunogi et al focused on a specific niche that their redesign would target, the purpose for Diokno et al's redesign is specifically for pelvic organ prolapse quantification [4]. Changes include adjustable top and bottom blades that are marked by centimeters and are thicker and rounded. The handle mechanism can allow the shortened bills to be expanded. In comparison to the Veda Scope, Diokno et al's speculum is closer in design to McDonagh et al's with the exception of elongation of the bills, the spring component, and sheaths. Both papers made minimal changes to the speculum. An interesting point of contention is between lengthening versus shortening the bills. In the physician interviews conducted, it was a general consensus that for certain populations (i.e. obese populations and women with pelvic organ prolapse), longer bills were more appropriate (Israel). Conversely, for children, a vaginal speculum with shorter bills was preferable (Hakim). Diokno et al's pilot study was conducted with 11 participants which had a low correlation coefficient at 0.088. However, Diokno et al concluded that the data favors the Diokno et al redesign. More research with a larger sample size ought to be conducted to determine efficacy of Diokno et al's design to other redesigns.

\section{Asiedu et al}

Asiedu et al engineered an entirely new device that would entirely replace the vaginal speculum while serving the same purpose as a speculum. Asiedu et al proposed a tubular device, approximately 2.5 centimeters in diameter, with a mini camera for image capturing and LEDs illuminating the vaginal cavity. Unlike the speculum redesigns, Asiedu et al's device is able to capture images of the cervix while simultaneously allowing for pap smears. The Veda Scope, McDonagh et al, and Diokno et al had proposed designs to increase visualization of the cervix and allow for samples to be taken with insertion of the tool done by a physician; however, Asiedu et al's compact, self-inserting colposcope addresses the problems with physical comfort and efficacy of the current bi-valve speculum design. Results from the Diokno et al 


\section{Columbia Undergraduate Research Journal}

clinical trials found that there was a $92.3 \%$ preference for the redesign over the bivalve design, as well as adequate cervix visualization, indicating that efficacy and comfort were not compromised for one another. The effectiveness of this substitute needs to be investigated further compared to other novel redesigns and the current bivalve speculum.

\section{Miscellaneous}

Due to the lack of strong connection of these papers compared to the rest, we labelled these articles as miscellaneous. This category of publications had two general overviews of the vaginal speculum and a paper focusing on the preferability of reusable and disposable specula using an environmental perspective. Regarding the historical context of the vaginal speculum, in a span of eight years, the two papers were published detailing the extensive history behind the speculum. In 2000, Sandelowski [46] discussed the context of the speculum coupled with commentary on how speculum usage could be a reclamation of power for women since, as stated in the introduction, the speculum was a tool of oppression of slave women. Essentially, Sandelowski cushions the history of the speculum with social implications of the speculum. Eight years later, Rossman [47] also provides a general overview and history of the vaginal bi-valve speculum like Sandelowski. However, Rossman centers the publication on previous attempts on redesigning the speculum, which have notably failed to receive traction in the medical setting due to physician reluctance to try new techniques and devices. No changes to the field have been discussed from Sandelowski to Rossman. Even more alarming is that there have been no overviews in the last 13 years on any changes to the vaginal speculum regarding women's health, despite there being a plethora of redesigns and pelvic examination improvement papers published in the past 20 years. This implies a disconnect between industry and academia which would explain the lack of presence of redesigns in the market as well as the stagnancy in research about the vaginal speculum in recent years. The latest publication about the vaginal speculum written by Donahue et al was to evaluate the carbon footprint between three vaginal specula: one acrylic and two stainless-steel. In 20 gynecologic exams, it was determined that the stainless-steel speculum 304 was environmentally preferred. Despite the study being unrelated to patient experience or increasing efficacy of the examination, the recent interest in the vaginal speculum is promising. With these publications in mind, more discussion needs to take place between academia and industry in order to facilitate progress for the vaginal speculum.

\section{Obesity}

In the introduction, it was noted that obese populations are often neglected by both industry and health providers, which is especially concerning in the context of a 


\section{Columbia Undergraduate Research Journal}

pelvic examination, which is a particularly intimate process. This patient population often faces stigma in the health field (Friedman). Not only is this population provided lower quality care during visits, but medical instruments aren't accommodating for them (Clarke; Friedman). This deters obese patients from visiting clinics and in turn, from having pelvic examinations (Pausé). Reasons for deterrence included health provider attitudes and treatment, issues concerning weight, and unsuitable equipment (Amy). Amy et al found that physicians typically did not have specula to accommodate obese women during their gynecological cancer examinations. Ahmed et al detail that the current bi-valve vaginal speculum is unsuitable in that physicians are still unable to view the cervix despite larger bills_-indicating that no industry efforts have been made to accommodate the obese population. In order to address this problem, practicing physicians regularly used condoms as a placeholder to prevent obstruction of the cervix for obese women (Israel). Furthermore, as detailed earlier, Freeman et al focused on the condom as a substitute sheath for the vaginal speculum to prevent vaginal walls from inhibiting the view of the cervix. It was mentioned throughout the publication that this method was mostly used for obese women. This makeshift solution suggests that there are no current medical devices nor research conducted to create medical care to suit obese populations.

\section{Conclusion}

Considering that physician interviews highlight the issues of Pederson/Graves bi-valve speculum use on the general patients, and more specifically obese patients, the lack of literature on the topic is concerning. Through further research outside of the literature review, there seem to have been many attempts to redesign the vaginal speculum, although these industry solutions are not evident in the current literature. Very few articles evaluate the effectiveness of novel designs, with the only novel design in literature being the Veda Scope. Thus, there seems to be a major disconnect between industry and academia. Without published, rigorous testing on these devices, physicians will not adopt these solutions, and patients will continue to receive the unsuitable current standard of care. For advancement to occur, the literature needs to more accurately reflect the current solutions. In addition, obese patients face unique obstacles regarding proper healthcare. Due to the high level of discomfort of the vaginal speculum, it has been proven that obese patients avoid and/or delay necessary examinations. In order to ensure that all patients have access to appropriate healthcare, more research must be conducted on the vaginal speculum and the pelvic examination procedure for obese patients.

\section{Bibliographic Notes}

1. See results section for further information 


\section{Columbia Undergraduate Research Journal}

2. A technique that is said to lead to quicker pelvic examinations and minimized discomfort for the patient. See footnote 3 for further information

3. Gynecologic interstitial brachytherapy is the implementation of catheters through the vagina, the perineum, or in and bordering a tumor

4. Pelvic Organ Prolapse Quantification is a method of standardizing the extent of prolapse of pelvic organs.

\section{WORKS CITED}

Ahmed, Syed M., Jeanne Parr Lemkau, and Sandra Lee Birt. "Toward Sensitive Treatment of Obese Patients in Primary Care." Family practice management 9.1 (2002): 25.

Amy, Nancy K., et al. "Barriers to routine gynecological cancer screening for White and African-American obese women." International journal of obesity 30.1 (2006): 147-155.

Asiedu, Mercy Nyamewaa, et al. "Design and preliminary analysis of a vaginal inserter for speculum-free cervical cancer screening." PloS one 12.5 (2017): e0177782.

Bates, Carol K., Nina Carroll, and Jennifer Potter. "The challenging pelvic examination." Journal of general internal medicine 26.6 (2011): 651-657.

Bridea Medical. "Speculum for Medical Use." Bridea Medical, 8 Feb. 2021, www.brideamedical.com/?v=7516fd43adaa.

Clarke, Megan A., et al. "Epidemiologic evidence that excess body weight increases risk of cervical cancer by decreased detection of precancer." Journal of Clinical Oncology 36.12 (2018): 1184.

Diokno, Ananias C., and German Borodulin. "A new vaginal speculum for pelvic organ prolapse quantification (POPQ)." International Urogynecology Journal 16.5 (2005): 384-388.

Donahue, Laura M., et al. "A comparative carbon footprint analysis of disposable and reusable vaginal specula." American journal of obstetrics and gynecology 223.2 (2020): 225-e1.

"Duke Team Reaches Milestone with Portable Cervical Cancer Screening Device."

Duke Global Health Institute, Duke, 14 Feb. 2017,

globalhealth.duke.edu/news/duke-team-reaches-milestone-portable-cervicalcancer-screening-device.

Freeman, Lisa. "Condom use to aid cervical visualization during speculum examination." Canadian Family Physician 64.4 (2018): 297-298.

Friedman, Asia M., et al. "Obese women's barriers to mammography and pap smear: the possible role of personality." Obesity 20.8 (2012): 1611-1617.

Gates, Elena, Personal Communication. 23 September 2020 


\section{Columbia Undergraduate Research Journal}

Hakim, Julia, Personal Communication. 1 October 2020

Hill, D. Ashley, and Georgine Lamvu. "Effect of lubricating gel on patient comfort during vaginal speculum examination: a randomized controlled trial." Obstetrics \& Gynecology 119.2 (2012): 227-231.

Hill, David A., Michael L. Cacciatore, and Georgine Lamvu. "Sheathed versus standard speculum for visualization of the cervix." International Journal of Gynecology \& Obstetrics 125.2 (2014): 116-120.

IPSOS Global Advisor. "Global Views On Healthcare.” IPSOS, 2018, www.ipsos.com/sites/default/files/ct/news/documents/201807/global_views_on_healthcare_2018_-_graphic_report_0.pdf.

Israel, Robert, Personal Communication. 24 September 2020

Jones, Christina L., et al. "Usability Study of a Novel, Self-Lighted, Disposable Speculum: Military Applications." Military medicine 178.4 (2013): e489-e492.

Kent, Chloe. "Does the Vaginal Speculum Need a Redesign? - Medical Technology: Issue 31: September 2020.” Medical Technology | Issue 31 | September 2020, 4

Sept. 2020, medical technology.nridigital.com/ medical_technology_sep20/ vaginal_speculum_red esign.

Kunogi, Hiroaki, Nanae Yamaguchi, and Keisuke Sasai. "Evaluation of a new bivalve vaginal speculum applicator design for gynecologic interstitial brachytherapy." Journal of contemporary brachytherapy 12.1 (2020): 27.

Longmore, Peter G. "Veda-scope: More comfortable than the bivalve speculum and cytologically equivalent." Australian and New Zealand journal of obstetrics and gynaecology 44.2 (2004): 140-145.

Mailach, Dayna. "LOTUS: Speculum Redesign.” Dayna Mailach, daynamailach.com/lotus-speculum-redesign.

Martinez, Gladys M., et al. "Receipt of pelvic examinations among women aged 1544 in the United States, 1988-2017." (2019).

Matschukat, Julia, and Maria Lalli. "Press - News." Ceek Women's Health, 19 Nov. 2020, ceekwomenshealth.com/ceek-news/category/Press.

National Ambulatory Medical Care Survey: 2015 State and National Summary Tables. U.S. Department of Health and Human Services, 2015, www.cdc.gov/nchs/data/ahcd/namcs_summary/2015_namcs_web_tables.p df.

Pausé, Cat. "Die another day: the obstacles facing fat people in accessing quality healthcare." Narrative inquiry in bioethics 4.2 (2014): 135-141.

Rossmann, Jenn Stroud. "Built to spec?: The vaginal speculum as a case study of inadequate design." (2008).

Sandelowski, Margarete. "This Most Dangerous Instrument': Propriety, Power, and the Vaginal Speculum." Journal of Obstetric, Gynecologic, \& Neonatal Nursing 29.1 (2000): 73-82. 


\section{Columbia Undergraduate Research Journal}

Seehusen, Dean A., et al. "Improving women's experience during speculum examinations at routine gynaecological visits: randomised clinical trial." bmj 333.7560 (2006): 171.

Taylor, Gina A., Deana McDonagh, and Michael J. Hansen. "Improving the pelvic exam experience: a human-centered design study." The Design Journal 20.sup1 (2017): S2348-S2362.

Thomas, Anna, et al. "A randomised controlled trial comparing a dilating vaginal speculum with a conventional bivalve speculum." Australian and New Zealand journal of obstetrics and gynaecology 41.4 (2001): 379-386.

Traub, Matthew. "Learn About HER-SPEC." Obp, 10 Nov. 2020, obpmedical.com/clickfree/.

Wright, Deborah, et al. "Speculum 'self-insertion': a pilot study." Journal of clinical nursing 14.9 (2005): 1098-1111.

Yona. "Speculum." Yona Care, yonacare.com/Speculum. 